\title{
Europa mit beschränkter Haftung
}

\section{Die Verabschiedung der EU-Richtlinie zur Umwelthaftung stockt. Wichtige Institutionen wie efwa das Europäische Parlament sind in sich gespalten, der Druck von Umweltverbänden und Industrie auf die Entscheidungsträger ist groß. Ein genauer Blick auf den Richtlinienentwurf erklärt, warum der jetz† ein Jahr davernde Prozess noch nicht beendet ist.}

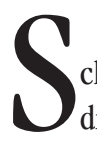
Von Benjamin Lünenbürger denen Umweltschäden auf. Die EU sieht seit längerem auf dem Gebiet der Umwelthaftung Handlungsbedarf. Im Frühjahr 2000 hat die EU-Kommission ein Weißbuch zur Umwelthaftung vorgelegt, auf das im Januar 2002 ein erster Richtlinienvorschlag folgte, der seitdem in den Gremien der EU zur Diskussion steht (1).

Die von der Kommission vorgeschlagene Richtlinie zur Umwelthaftung will bestehende Haftungslücken für bestimmte Umweltschäden innerhalb der EU schließen. Umweltschäden im Sinne des Richtlinienvorschlages sind nachteilige Veränderungen der biologischen Vielfalt in ausgewiesenen Schutzgebieten, von Gewässern sowie von Böden. Die Richtlinie und die in ihr vorgesehene Gefährdungshaftung kommt nicht zur Anwendung, wenn die Bestimmungen von Betriebsgenehmigungen eingehalten sind oder der Schaden nach wissenschaftlichen und technischen Erkenntnissen nicht bekannt war. Ein Haftungsanspruch bei Personen- und Sachschäden und für Altlasten kann über die Richtlinie nicht begründet werden.

In einem gesonderten Anhang zur Richtlinie werden die im Haftungsfall erforderlichen Sanierungs- und Ausgleichsmaßnahmen geregelt. Kann der Verursacher von Umweltschäden nicht identifiziert oder haftbar gemacht werden, oder verfügt er nicht über ausreichende finanzielle Mittel, so sind die zuständigen Behörden verpflichtet, erforderliche Sanierungsmaßnahmen selbst durchzuführen und dafür aufzukommen. Qualifizierte Einrichtungen wie zum Beispiel Umweltverbände haben das Recht, Behörden zum Tätigwerden aufzufordern. Ausdrücklich wird in der Richtlinie den Mitgliedsstaaten die Möglichkeit gelassen, auf einzelstaatlicher Ebene strengere Vorschriften zu erlassen.

\section{Theoretische Vorteile}

Der Richtlinienentwurf strebt eine Stärkung des Umwelthaftungsrechts an. Die umweltökonomische Literatur unterscheidet zwei Haftungsregeln, die im Idealfall gleichermaßen über die Schaffung eines optimalen Vorsorgeniveaus zur Vermeidung von Schäden führen. Bei der Gefähr dungshaftung haftet der Schädiger grundsätzlich und bezieht in der Theorie deshalb nicht nur etwaige Vorsorgeanstrengungen in sein wirtschaftliches Kalkiul mit ein, sondern auch die Umweltschäden, für die er haften muss. Bei der Verschuldenshaftung haftet der Schädiger nur, wenn Verschuldensstandards verletzt sind. Sind die Standards richtig gesetzt, so kann ein optimales Vorsorgeniveau erreicht werden. Sind die Annahmen des Grundmodells nicht erfüllt, so führen beide Haftungsregeln zu unterschiedlichen Ergebnissen. Die Gefährdungshaftung ist bei Schadensdiskontierung nicht robust, die Verschuldenshaftung schneidet in diesem Punkt zwar besser $a b$, hängt aber entscheidend von optimal gesetzten Verschuldensstandards ab. Außerdem ist die Verschuldenshaftung nicht vereinbar mit dem Verursacherprinzip, denn Schädiger werden zwar angehalten ein volkswirtschaftlich optimales Vorsorgeniveau zu betreiben, müssen aber bei Einhaltung der Standards für etwaige Umweltschäden nicht aufkommen. Verbleibende Schäden werden von den Geschädigten getragen. Die in der Richtlinie angestrebte Schließung von Haftungslücken ist aus umweltökonomischer Sicht zu begrüßen, denn nur bei eventueller Haftung für Umweltschäden besteht ein Vorsorgeanreiz.

\section{Defizite nur teilweise behoben}

Ein großes Defizit im bisherigen Haftungsrecht sieht die Literatur in der mangelnden Haftung für Umweltschäden, die nicht individualisierbare Rechtsgüter betreffen. Der Richtlinienentwurf begründet eine Haftung für einige dieser reinen Umweltschäden und stellt sich der Aufgabe, Grund- sätze für eine Kompensation von Umweltschäden zu formulieren. Dabei gilt das Prinzip der Naturalrestitution; alternativ kommen aber auch Ausgleichsmaßnahmen in Frage. Außerdem müssen zwischenzeitliche Verluste kompensiert werden. In diesem Zusammenhang können ökonomische Bewertungsmethoden zur Anwendung kommen. Die Grundsätze der Sanierung lehnen sich an die Regelungen des US-amerikanischen Oil Pollution Act an.

Durch die zahlreichen Haftungsausschlüsse, beispielsweise soll bei Tankerunfällen und nuklearen Risiken die Richtlinie explizit keine Anwendung finden, oder durch Nichtberücksichtigung bei der Liste der gefährlichen Tätigkeiten im Anhang, etwa bei einigen Tätigkeiten mit gentechnisch veränderten Organismen, bleiben viele bestehende Haftungslücken offen. Ein volkswirtschaftlich effizientes Vorsorgeniveau ist deshalb zukünftig in diesen Fällen nicht zu erwarten. Zwar gilt im Richtlinienentwurf zunächst die Gefährdungshaftung, doch wird in vielen Fällen die Haftung letztlich doch vom Verschulden abhängig gemacht. Eine verursachungsgemäße Anlastung von Umweltschäden findet in diesen Fällen deshalb nicht statt.

Die nächsten Monate werden zeigen, ob bei den Beratungen im Europäischen Parlament und im Europa-Rat der Richtlinienentwurf weiter abgeschwächt wird oder ob das Verursacherprinzip im EU-Umweltrecht gestärkt werden kann. Es bleibt abzuwarten ob das ewige Thema Umwelthaftung - ein erstes Grünbuch wurde bereits 1993 von der Kommission vorgelegt - auf EU-Ebene bald abgeschlossen ist.

\section{Anmerkungen}

(1) Europäische Kommission: Weißbuch zur Umwelthaftung, KOM (2000) 66 endg., Brüssel 2000 sowie Europäische Kommission: Vorschlag für eine Richtlinie des Europäischen Parlaments und des Rates über Umwelthaftung betreffend die Vermeidung von Umweltschäden und die Sanierung der Umwelt, KOM (2002) 17 endgültig, Brüssel 2002.

\section{Der Autor}

Benjamin Lünenbürger promoviert am Graduiertenkolleg für Umwelt- und Ressourcenökonomik der Universitäten Heidelberg und Mannheim Kontakt: Interdisziplinäres Institut für Umweltökonomie, Bergheimer Str. 20, D-69115 Heidelberg, E-Mail: lvenenbuerger@eco.uni-heidelberg.de 
(c) 20I0 Authors; licensee IÖW and oekom verlag. This is an article distributed under the terms of the Creative Commons Attribution Non-Commercial No Derivates License (http://creativecommons.org/licenses/by-nc-nd/3.o/), which permits unrestricted use, distribution, and reproduction in any medium, provided the original work is properly cited. 